\title{
Impact of HIV-1 CRF55_01B infection on the evolution of CD4 count and plasma HIV RNA load in men who have sex with men prior to antiretroviral therapy
}

\section{Lan Wei}

Shenzhen Center for Disease Control and Prevention

Hao Li

Shenzhen Center for Disease Control and Prevention

Xing Lv

Shenzhen Center for Disease Control and Prevention

\section{Chenli Zheng}

Shenzhen Center for Disease Control and Prevention

\section{Guilian Li}

Shenzhen Center for Disease Control and Prevention

\section{Zhengrong Yang}

Shenzhen Center for Disease Control and Prevention

\section{Lin Chen}

Shenzhen Center for Disease Control and Prevention

\section{Xiaoxu Han}

The First Hospital of China Medical University: The First Affiliated Hospital of China Medical University

Huachun Zou

Sun Yat-sen University School of Public Health

\section{Yanxiao Gao}

Sun Yat-Sen University School of Public Health

\section{Jinquan Cheng}

Shenzhen Center for Disease Control and Prevention

Hui Wang

Shenzhen Center for Disease Control and Prevention

Jin Zhao ( $\square$ zhaoj@szcdc.net )

Shenzhen Center for Disease Control and Prevention

\section{Research}


Keywords: Disease progression, circulating recombinant form, CD4 count, viral load

Posted Date: April 9th, 2021

DOI: https://doi.org/10.21203/rs.3.rs-390294/v1

License: (c) (1) This work is licensed under a Creative Commons Attribution 4.0 International License. Read Full License

Version of Record: A version of this preprint was published at Retrovirology on August 16th, 2021. See the published version at https://doi.org/10.1186/s12977-021-00567-z. 


\section{Abstract}

Background CRF55_01B is a newly identified HIV-1 circulating recombinant form originated from MSM in China. However, its impact on the disease progression and transmission risk has not been investigated. This study aimed to determine the impact of CRF55_01B infection on viral dynamics and immunological status, so as to provide implications for future prevention, treatment, or target interventions. Linear mixed effect models were applied to evaluate CD4 cell count decline and viral load increase by subtype.

Results Of the 3418 blood samples, 1446 (42.3\%) were CRF07_BC, 1169 (34.2\%) CRF01_AE, 467 (13.7\%) CRF55_01B, 249 (7.3\%) type B, and 87 (2.5\%) other subtypes (CRF_08BC, CRF_01B, C). CRF55_01B had replaced subtype B as the third predominant strain since 2012 in Shenzhen, China. CRF55_01B-infected MSM showed lower median of CD4 count than CRF07_BC-infected MSM (349.5 [IQR, 250.2 474.8] vs 370.0 [IQR, 278.0 501.0], $\mathrm{P}<0.05)$. CRF55_01B infection was associated with slower loss of CD4 count than CRF01_AE (13.6 vs 23.3 [cells/ $\mu \mathrm{L}]^{1} / 2 /$ year, $\mathrm{P}<0.05$ )among MSM with initial CD4 count of 200 350 cells/ $\mu \mathrm{L}$. On the other hand, those infected with CRF55_01B showed higher median plasma HIV RNA load (5.4 [IQR, 5.0 5.9]) than both CRF01_AE (5.3 [IQR, 4.8 5.7], P<0.05) and CRF07_BC (5.0 log10 [IQR, 4.5 5.5], $P<0.001)$ at the initiation of antiretroviral therapy. Furthermore, the annual increasing rate of viral load for CRF55_01B infection was significantly higher than that of CRF07_BC (2.0 vs $0.7 \log 10$ copies/ml/year, $\mathrm{P}<0.01)$.

Conclusions The relatively lower CD4 count and faster increase of plasma HIV RNA load of CRF55_01Binfected MSM without antiretroviral therapy suggest that CRF55_01B may lead to longer asymptomatic phase and higher risk of HIV transmission. Strengthened surveillance, tailored prevention strategies and interventions, and in-depth research focusing on CRF55_01B are urgently needed to forestall potential epidemic.

\section{Introduction}

HIV has very high genetic variability and diversity. To date, two main types, nine subtypes, 89 HIV circulating recombinant forms (CRFs) and multiple unique recombinant forms (URFs) had been recognized worldwide [1]. In Asia, the predominant genotypes are subtype B and C, CRF01_AE and their recombinants [2]. With the increasing trend of HIV prevalence among men who have sex with men (MSM) in China in the past decade [3], the three predominant genotypes co-circulated and rapidly mixed, leading to inevitable generation of new CRFs. Previous studies implied that CRFs accounted for more than $90 \%$ of all HIV-1 infection in China, and new CRFs played an increasing role in the nationwide or regional HIV pandemic $[4,5]$.

CRF55_01B, a CRF derived from CRF01_AE and B and circulating predominantly among MSM, was identified in 2012 in China [3]. Surveillance data indicated that CRF55_01B has been the third important epidemic CRFs since CRF07_BC and CRF08_BC were discovered in China in the 1990s [3], and has brought about an outbreak among MSM in Shenzhen in 2013 [6]. Previous studies implied the origin time 
of CRF55_01B in China was around 2001, and first infected case was found in Shenzhen in 2007 [6]. Existing literature indicated that the prevalence of HIV-1 CRF01_AE and subtype B in Shenzhen had gradually decreased from $50 \%$ and $37.5 \%$ to $32.3 \%$ and $5.7 \%$ from 2006 to 2012 , respectively. In contrast, the prevalence of CRF07_BC and CRF55_01B had rapidly increased from $25 \%$ and $0 \%$ in 2006 to $43.2 \%$ and $16.0 \%$ in 2012 , respectively [7].

HIV-infected individuals without antiretroviral therapy are clinically characterized by loss of CD 4 T-cell and rise of plasma HIV RNA load, which could result in increased risks for opportunistic infections and development of AIDS and AIDS-related deaths. Thus, both CD4 T-cell counts and plasma HIV RNA are important prognostic markers of progression to AIDS and serve as main indicators for initiation of antiretroviral therapy. Clinical studies revealed that HIV-1 subtype was closely related to HIV transmission and disease progression [8-11]. As a newly emerging strain, the evolutionary characteristics of CRF55_01B has been identified; nevertheless, its impact on the evolution of CD4 count and plasma HIV RNA load, has not been documented. In the present study, we aimed to better understand the impact of CRF55_01B infection on viral dynamics and immunological status and its transmission risk among those infected MSM prior to antiretroviral therapy, so as to provide implications for future prevention, treatment, or target interventions.

\section{Results}

\section{Demographic characteristics}

From 2005 to 2015, blood samples were collected from 3418 MSM at diagnosis of HIV infection in Shenzhen, with genotype distribution as follows: 1446 (42.3\%) CRF07_BC, 1169 (34.2\%) CRF01_AE, 467 (13.7\%) CRF55_01B, 249 (7.3\%) subtype B, and 87 (2.5\%) other subtypes (CRF_08BC, CRF_01B, C, and others) (Figure 1). Of note, we found that CRF55_01B had significantly expanded during the past years $(P<0.05)$, and had replaced subtype $B$ as the third predominant strain since 2012 in Shenzhen, China. By excluding the other subtypes and the infections which were more than one year prior to sampling, there were 1792 recent infection for CRF07_BC, CRF01_AE, and CRF55_01B. Demographic characteristics of the 1792 MSM are shown in table 1.

\section{CD4 T-cell count and plasma HIV RNA load}

Among the 1792 samples for the three main subtypes, the mean genetic distance was $0.045 \pm 0.003$ for CRF01_AE, 0.018 \pm 0.001 for CRF07_BC, and 0.022 \pm 0.002 for CRF55_01B. CRF55_01B-infected MSM showed a significantly lower median CD4 count than CRF07_BC-infected MSM at diagnosis of HIV infection (349.5 [IQR, 250.2 474.8] vs 370.0 [IQR, 278.0 501.0], P<0.05), as well as at initiation of cART (224.0 [IQR, 165.2 283.0] vs 265.0 [IQR, 198.0 348.0], P<0.01) (Figure 2). However, the median CD4 count of CRF55_01B-infected MSM was not significantly different from that of CRF01_AE-infected MSM at diagnosis of HIV infection (349.5 [IQR: 250.2 474.8] vs 335.0 [IQR: 237.0 464.0], $\mathrm{P}=0.352$ ) and at initiation of cART (224.0 [IQR, 165.2 283.0] vs 203.0 [IQR, 130.5 287.0], P=0.154). In contrast, CRF55_01B-infected MSM was associated with a significantly higher median plasma HIV RNA load (5.4 
$\log _{10}$ copies/ml; IQR, 5.0 5.9) than CRF01_AE-infected MSM (5.3 $\log _{10}$ copies/ml; IQR, 4.8 5.7, P<0.05) and CRF07_BC (5.0 $\log _{10}$ copies/ml; IQR, 4.5 5.5, P<0.01) at the initiation of CART (Figure 2). In addition, those infected with CRF55_01B showed significantly higher plasma HIV RNA load (4.7 $\log _{10}$ copies/ml; IQR, 4.1 5.3) than those infected with CRF07_BC (4.3 $\log _{10}$ copies/ml; IQR, 3.7 4.8, P<0.01) at diagnosis, although similar to those infected with CRF01_AE (4.6 $\log _{10}$ copies/ml; IQR, 4.1 5.1, P=0.695).

\section{Change trend of CD4 T-cell count and plasma HIV RNA load}

A total of 753 MSM were included for estimate and comparison of the declining rates of CD4 count by subtype, and 199 MSM were included for analysis of the increasing rates of plasma HIV RNA load by subtype. Demographic characteristics and time interval between the two measurements of CD4 count and viral load were presented in Supplementary table 1 and 2 . Stratified analyses were conducted according to the initial CD 4 count at diagnosis as different initial CD4 counts represent different status of disease progression of the patients. Table 2 showed the estimated declining rates of CD 4 counts by subtype. Specifically, for the group with initial CD 4 counts between 200 and 350 cells/ $\mu \mathrm{L}$, infection with CRF55_01B was associated with a significantly lower declining rate ([cells $/ \mu \mathrm{L}]^{1 / 2} /$ year) than with CRF01_AE (13.4 [95\%Cl, 8.2 18.6] vs 22.9 [95\%Cl, 15.8 30.0], P <0.05), while similar to CRF07_BC (13.4 [95\%Cl: 8.2 18.6] vs 10.8 [95\% Cl, 8.2 13.4], $\mathrm{P}=0.383$ ). However, no significant difference was found for the other group with initial CD4 value above 350 cells/ $\mu \mathrm{L}$. Linear mixed effect model results also showed that the declining rate of CD4 count for CRF55_01B-infected MSM was 10.3 [cells/ $\mu \mathrm{L}]^{1 / 2} /$ year $(95 \% \mathrm{Cl}$, 0.7 19.9, $P<0.05$ ) lower than that for CRF01_AE-infected MSM among the patients with CD4 counts of 200 350 cells/ $\mu \mathrm{L}$ at HIV diagnosis, with adjustments for age at diagnosis, ethnicity, marital status, and year of HIV diagnosis.

In general, for all MSMS with initial CD4 count of 200 cells/ $\mu \mathrm{L}$ or above, CRF55_01B infections caused a significantly higher increasing rate of plasma HIV RNA load than CRF07_BC infections $\left(2.0 \log _{10}\right.$ copies/ml [95\% Cl, 1.1 2.9] vs $0.7 \log _{10}$ copies/ml [95\% Cl, 0.5 0.9], $\mathrm{P}<0.01$ ), while not significantly different from infection with CRF01_AE (1.4 $\log _{10}$ copies/ml [95\%Cl, 0.8 2.0], P=0.324) (Table 3). Furthermore, the model with random effect also showed CRF55_01B infections were $1.2 \log _{10}$ copies/ml/year higher increasing rate of viral load than CRF07_BC infections after adjustment for age at diagnosis, ethnicity, marital status, and year of HIV diagnosis, although not significantly different for the group with initial CD4 count as 350 cells/ $\mu \mathrm{L}$ or above.

\section{Discussion}

Our study found that CRF55_01B, a newly emerged CRF of HIV-1, had become the third predominant strain in recent years in Shenzhen, China. With long-term surveillance data of the past 10 years in Shenzhen, this study for the first time revealed the impact of infection with CRF55_01B on the immunological progression and viral replication in the CART-naive MSM and compared it with other two dominant subtypes. We found that CRF55_01B-infected MSM had significantly lower CD4 count than 
CRF07_BC-infected MSM both at diagnosis and the initiation of cART. Furthermore, CRF55_01B showed significantly slower loss of CD4 count than CRF01_AE among the patients with initial CD4 count of 200 350 cells/ $\mu \mathrm{L}$. Meanwhile, CRF55_01B-infected MSM showed significantly higher plasma HIV RNA load than MSM infected with CRF01_AE or CRF07_BC at the initiation of cART. Moreover, infection with CRF55_01B was associated with a significantly faster increase of plasma HIV RNA load than infection with CRF01_AE and CRF07_BC, especially among the patients with initial CD4 count of 200 350 cells/ $\mu \mathrm{L}$.

CRF55_01B had overtaken subtype B as the third predominant strain in Shenzhen since 2012. Recent studies indicated that CRF55_01B had rapidly spread and epidemic from Guangdong-Shenzhen to other provinces of China with prevalence ranging from 1.5\% to $12.5 \%$ since 2010; of note, the highest prevalence was reported in Guangdong, China [12-14]. Our study revealed that infection with CRF55_01B had significantly slower loss of CD4 count than infection with CRF01_AE among those with the initial CD4 count between 200 and 350 cells/ $\mu \mathrm{L}$. Slow depletion of CD4 T-cell usually indicates slow immunological progression and less impairment to human system, which may translate to a prolonged asymptomatic state and increased risk for onward HIV transmission [15]. Although the significant difference was only observed in patients with initial CD4 count between 200 and 350 cells/ $\mu \mathrm{L}$, it was justifiable because previous study demonstrated that only baseline CD 4 count below 350 cells/ $\mu \mathrm{L}$ was significantly associated with rapid decline of CD4 count [16]. A recent nationwide HIV molecular epidemiologic survey by China CDC discovered that people infected with CRF01_AE cluster 5 had significantly higher CD4 count than those infected with CRF01_AE cluster 4 [17]; meanwhile, our unpublished data based on general population found that CRF55_01B-infected MSM had even higher CD4 count than those infected with CRF01_AE cluster 5, which may be attributed to the slower loss of CD4 count caused by infection with CRF55_01B than with CRF01_AE. More studies are warranted to confirm the findings.

It is well documented that patients infected with CRF01_AE had higher plasma HIV RNA load than those with CRF07_BC [18]. A previous cohort study suggested that CRF07_BC was associated with relatively lower plasma HIV RNA load, which could probably be explained by its poorer protease-mediated processing and slower viral maturation processes [19]. However, our study found that patients infected with CRF55_01B had even higher plasma HIV RNA load than those infected with CRF01_AE at the initiation of cART, and had faster increasing rate than those infected with CRF01_AE during the rapid disease progression period. Higher viral load and faster increasing rate of viral load represent higher viral replication capacity, and may increase the risk of HIV transmission [20]. Thus, the faster increase of viral load together with the relatively slower loss of CD4 T-cell may help to explain the recent surging dominance and continued expansion of CRF55_01B among MSM in China. As the published data on CRF55_01B were only limited to the genomic and evolutionary characteristics, information on its impact on the disease progression has rarely been reported, making it difficult to compare our findings with studies from other regions.

Consistent with a recent study from Guangdong, our study found CRF07_BC and CRF01_AE were the two predominant subtypes in Shenzhen [21]. CRF01_AE, first imported to China in 1994 [22], now has become 
a main strain in MSM in several cities of China [23-25], and highly dynamic in Southeast Asia [26]. The predominant HIV-1 epidemic strain in the Chinese MSM has recently changed from subtype B of USEuropean origin in 2005 to CRF01_AE in 2012 [27]. Previous study suggested that CRF01_AE strain with a high frequency of CXCR4-tropism circulating in MSM population might cause a severe loss of CD4 count and speed up the disease progression, compared to CRF07_BC and subtype B strains [8]. Other studies also found that CRF01_AE strains was associated with a higher annual rate of CD4 loss and accelerated disease progression than the non-CRF01_AE strains $[28,29]$.

Infection with CRF55_01B was associated with lower CD4 count than infection with CRF07_BC both at diagnosis and the initiation of CART in this study. CRF07_BC, the generation of Thailand variant of subtype B and Indian subtype C, was initially found among IDUs in southwest China in the 1990's and then spread to other areas [30-32]. It was first found to be transmitted to MSM since 2004 and has become one of the most prevalent strains for over ten years [13,33]. A study from Taiwan suggested that CRF07_BC among IDUs was associated with relatively slower depletion of CD4 count, which was attributed to the 7-amino acid deletion observed in p6 of CRF07_BC [34].

It is also noteworthy that previous molecular epidemiologic analysis demonstrated that CRF55_01B might be originated from MSM in Shenzhen and later transmitted to heterosexual population, and then spread to other regions of China through the migrant MSM $[6,14]$. As the largest immigration city in China, Shenzhen is a popular gathering place for migrant MSM from all over the country, Shenzhen would serve an important hub in the ongoing outspreading of CRF55_01B to other regions in China as well as other countries. A recent report of CRF55_01B infection in Hangzhou might be a prelude of outbreak in the central eastern China in the near future [8]. What's more, several new unique recombinant strains (e.g., SZ44LS7251 in Shenzhen) derived/originated from CRF55_01B and CRF07_BC were reported in southern China $[5,35,36]$, which may further complicate the genomic structures and give rise to super-infections or co-infections in the MSM community. All these potential consequences together pose serious challenges to the prevention and control of HIV infection, as well as the treatment and vaccine development [37]. Therefore, in-depth study and timely prevention and control of CRF55_01B infection are needed for both treatment strategies and prevention of potential epidemic.

There are several limitations worth noting. First, our study lack the estimated date of seroconversion of infected MSM, a common limitation in studies on disease progression [38]. Thus, the change rates for CD4 count and plasma HIV RNA load were not observed from the time of infection, but from the time of diagnosis. Nevertheless, we had adjusted the estimates by divide the dataset into 3 subsets with different initial level of CD4 counts, which could provide a rough proxy for different stages of disease progression. Secondly, this study only obtained two determinations of CD4 count and plasma HIV RNA load at diagnosis and the initiation of CART; thus, it is difficult to evaluate the dynamic change over time. Further study with more repeated measurements would be desirable. Also, the clinical outcomes should be monitored. Thirdly, the sample size for MSM with measurement on viral load at diagnosis was relatively small, which may lead to limited statistical power. Thus, future cohort studies with a larger sample size and more repeated measurements are needed to validate our findings. 


\section{Conclusions}

This study elucidated the pathogenic, immunologic and virological characteristics of HIV-1 CRF55_01B in infected MSM who were prior to cART. The relatively slower loss of CD4 count together with faster increase of plasma HIV RNA load suggest that CRF55_01B may prolong the asymptomatic phase and increase the risk of HIV transmission, which would favor further rise and potential epidemic in future. Our findings are helpful for understanding the disease progression and the recent surge of CRF55_01B among MSM in China, providing significant implications for future prevention, treatment, or target interventions to forestall potential epidemic and outspreading of CRF55_01B to other areas within China or outside the country.

\section{Methods}

\section{Study subjects}

HIV-1-infected MSM who were newly diagnosed with recent HIV infection and had been living in Shenzhen from 2005 to 2015 were screened according to the following conditions: 1) HIV-1 infection confirmed by Western blot (HIV blot 2.2, MP Diagnostics, Singapore); 2) HIV transmission through maleto-male sex; 3) prior to CART; 4) pol genes being sequenced and phylogenetically identified as CRF55_01B, CRF01_AE or CRF07_BC. Recent HIV infection was defined that the fraction of ambiguous nucleotides was lower than $0.5 \%$ in $\mathrm{po} /$ genes, which provides strong evidence against an infection event $<1$ year prior to sampling [39]. Written informed consent was obtained from all the participants recruited in this project, and the demographic data (such as gender, age, marital status, etc.) were also collected through face-to-face questionnaire interview. The protocol for enrollment of MSM in this study was approved by the Medical Ethics Committee of Shenzhen Center for Disease Control and Prevention (CDC).

Samples were collected at diagnosis and initiation of cART from patients seeking HIV care at Shenzhen Center for Disease Control and Prevention and the Third People's Hospital of Shenzhen. Each blood sample was collected in two EDTA- Vacutainer tubes with $5 \mathrm{ml} /$ tube (Becton and Dickinson Company, USA). One tube of whole blood sample was for quantification of CD4 count; and the other tube was centrifuged at $1500 \mathrm{~g}$ for $10 \mathrm{~min}$ at room temperature to separate plasma and buffy coat. Plasma was frozen in multiple aliquots at $-80^{\circ} \mathrm{C}$ until use.

\section{Phylogenetic Analysis}

HIV-1 genome RNA was extracted from stored plasma specimens using the QIAmp Viral RNA Mini kit (Qiagen, Valencia, CA, USA) as manufacturer's instructions. Fragment of $p o /$ were amplified by reverse transcription and nested PCR and then sequenced. Each sequence was blasted through the Los Alamos HIV-1 database, and checked for the existence of ambiguous nucleotides. The resulting gene fragment sequences were aligned with reference sequences to determine the subtypes while the phylogenetic tree was constructed by the neighbor-joining method implemented by MEGA version 7.0. Mean genetic 
distance of CRF01_AE, CRF07_BC and CRF55_01B strains was calculated under P-distance model (bootstrap value=500) using MEGA 7.0.

\section{CD4 T-Cell count and viral load assay}

CD4 count were measured in our laboratory by flow cytometry (FACS Calibur, Becton and Dickinson BD Company, USA) within 24h according to the instructions of BD Tritest CD4/CD8/CD3 (FITC/PE/PerCP, Becton and Dickinson Company, USA). HIV-1 genome RNA was extracted from stored plasma specimens using the HIV-1 nucleic acid testing of fluorescent PCR kit (Daan Gene, Guangzhou, China). PCR was performed on $\mathrm{ABI} 7500$ with quality control and calibrator, and viral load was automatically calculated as manufacturer's instructions. The linear detection range was $5.0 \times 10^{2}$ to $1.0 \times 10^{8} \mathrm{copies} / \mathrm{ml}$.

\section{Statistical analysis}

Demographic information of infections with different subtypes was tested for statistical difference by Chi-square test ( $\chi^{2}$-test) or Fisher's exact test where applicable. Plasma HIV-1 RNA load and CD 4 counts of patients infected with different HIV-1 subtypes (CRF01_AE, CRF07_BC, and CRF55_01B) were described using median and interquartile range (IQR). Kruskal-Wallis test (multiple independent samples nonparametric test) and Mann-Whitney test (two independent samples nonparametric test) were used to compare the CD4 counts and viral load among patients infected with different subtypes.

As previous studies suggested that serial CD4 observations decayed approximately linearly on the square root scale, square root transformation of CD4 value was applied to linearize the changes from the time of diagnosis to CART initiation in this study [10]. $\log _{10}$-transformation of viral load was utilized to linearize the change rate over time as well. Multilevel linear mixed effect models was used to compare the annual rate of CD4 cell decline and viral load increase. The final models adjusted for age at HIV diagnosis, ethnicity, marital status, and year of HIV diagnosis. As the date of infection was not known in this study, a random effect of the initial CD4 cell count was included in the models. Year of HIV diagnosis was also include as a categorical random effect to account for the random deviations of the criteria for CART initiation in the past years. Various studies on the decline of CD4 cell counts suggest those first CD 4 cell measurement made in the acute infection phases should be excluded. In this study, those with first CD 4 cell count lower than 200 cells/ $\mu \mathrm{L}$ or only have one measurement of CD 4 cell count were excluded from the analysis for comparison of CD 4 cell count declining and viral load increase by subtype.

All statistical tests were 2-tailed, probability value $<0.05$ were considered statistically significant. All statistical analyses were performed in R (version 3.4.4, Foundation for Statistical Computing, Vienna, Austria).

\section{List Of Abbreviations}

CRFs, circulating recombinant forms 
URFs, unique recombinant forms

MSM, men who have sex with men

$\mathrm{CDC}$, center for disease control and prevention

IQR, interquartile range

\section{Declarations}

\section{Ethics approval and consent to participate}

This study was approved by the Medical Ethics Committee of Shenzhen Center for Disease Control and Prevention. Written informed consent was obtained from all the participants recruited in this study

\section{Consent for publication}

Not applicable.

\section{Availability of data and materials}

The datasets supporting the conclusions of this article are included within the article and its additional files.

\section{Competing interests}

The authors report no conflicts of interest for this study.

\section{Funding}

This work was supported by the National Natural Science Foundation of China (81573211), Shenzhen San-Ming Project of Medicine in Shenzhen (SZSM201811071, SZSM201512029), Shenzhen Municipal Technological Project (JCYJ20160331173336891), Medical Scientific Research Foundation of Guangdong Province of China (A2019474), and Shenzhen Health and Family Planning System Research Project (SZGW201810003, SZGW2017006).

\section{Acknowledgments}

We thank Chiyu Zhang and Ping Zhong for assistance with study design and data analysis.

\section{Author's contributors}

JZ and HW conceived the work. LW, ZY, CL, JC, JZ, and HW designed the study. LW conducted the analysis and drafted the manuscript. HL contributed to the writing and data analysis. XL ran field investigations and participated in data management and interpretation. $\mathrm{HL}, \mathrm{CZ}$ and $\mathrm{GL}$ performed the experiments. HZ, YG contribute to the data analysis and writing. XH, HZ, ZY, JC, JZ and HW reviewed and 
edited the manuscript. All authors have seen and approved the final version of the manuscript for publication.

\section{References}

1. UNAIDS. Global AIDS Update. UNAIDS; 2017.

2. Oyomopito RA, Li PC, Sungkanuparph S, et al. Evaluating immunologic response and clinical deterioration in treatment-naive patients initiating first-line therapies infected with HIV-1 CRF01_AE and subtype B. J Acquir Immune Defic Syndr 2013; 62(3):293-300.

3. Zhang L, Chow EP, Jing J, et al. HIV prevalence in China: integration of surveillance data and a systematic review. Lancet Infect Dis 2013; 13(11):955-63.

4. Li X, Li W, Zhong P, et al. Nationwide Trends in Molecular Epidemiology of HIV-1 in China. AIDS Res Hum Retroviruses 2016; 32(9):851-9.

5. Jia D, Zhao J, Li T, et al. Near Full-Length Genomic Sequences of Two Novel HIV-1 Recombinant Forms Identified in Shenzhen, China. AIDS Res Hum Retroviruses 2017; 33(1):82-6.

6. Wei $\mathrm{C}$, Yan $\mathrm{H}$, Yang $\mathrm{C}$, et al. Accessing HIV testing and treatment among men who have sex with men in China: a qualitative study. AIDS Care 2014; 26(3):372-8.

7. Zhao J, Chen L, Chaillon A, et al. The dynamics of the HIV epidemic among men who have sex with men (MSM) from 2005 to 2012 in Shenzhen, China. Sci Rep 2016; 6:28703.

8. Zhao J, Cai W, Zheng C, et al. Origin and outbreak of HIV-1 CRF55_01B among MSM in Shenzhen, China. J Acquir Immune Defic Syndr 2014; 66(3):e65-7.

9. Taylor BS, Sobieszczyk ME, McCutchan FE, Hammer SM. The challenge of HIV-1 subtype diversity. N Engl J Med 2008; 358(15):1590-602.

10. Touloumi G, Pantazis N, Pillay D, et al. Impact of HIV-1 subtype on CD4 count at HIV seroconversion, rate of decline, and viral load set point in European seroconverter cohorts. Clin Infect Dis 2013; 56(6):888-97.

11. Kuritzkes DR. HIV-1 subtype as a determinant of disease progression. The Journal of infectious diseases 2008; 197(5):638-9.

12. Gan M, Zheng S, Hao J, et al. The prevalence of CRF55_01B among HIV-1 strain and its connection with traffic development in China. Emerg Microbes Infect 2021; 10(1):256-65.

13. Li Z, Liao L, Feng Y, et al. Trends of HIV subtypes and phylogenetic dynamics among young men who have sex with men in China, 2009-2014. Sci Rep 2015; 5:16708.

14. Han X, Takebe Y, Zhang W, et al. A Large-scale Survey of CRF55_01B from Men-Who-Have-Sex-withMen in China: implying the Evolutionary History and Public Health Impact. Sci Rep 2015; 5(1).

15. Yates A, Stark J, Klein N, Antia R, Callard R. Understanding the slow depletion of memory CD4+ T cells in HIV infection. PLoS Med 2007; 4(5):e177.

16. Chaiyasin N, Sungkanuparph S. Rate of CD4 Decline and Factors Associated with Rapid CD4 Decline in Asymptomatic HIV-Infected Patients. J Int Assoc Provid AIDS Care 2016; 15(1):3-6. 
17. Song H, Ou W, Feng Y, et al. Disparate impact on CD 4 T cell count by two distinct HIV-1 phylogenetic clusters from the same clade. Proc Natl Acad Sci U S A 2019; 116(1):239-44.

18. Li X, Xue Y, Cheng H, et al. HIV-1 Genetic Diversity and Its Impact on Baseline CD4+T Cells and Viral Loads among Recently Infected Men Who Have Sex with Men in Shanghai, China. PLoS One 2015; 10(6): $\mathrm{e} 0129559$.

19. Huang SW, Wang SF, Lin YT, et al. Patients infected with CRF07_BC have significantly lower viral loads than patients with HIV-1 subtype B: mechanism and impact on disease progression. PLoS One 2014; 9(12):e114441.

20. Attia S, Egger M, Muller M, Zwahlen M, Low N. Sexual transmission of HIV according to viral load and antiretroviral therapy: systematic review and meta-analysis. AIDS 2009; 23(11):1397-404.

21. Yang $C$, Liu S, Zhang $T$, et al. Transmitted antiretroviral drug resistance and thumb subdomain polymorphisms among newly HIV type 1 diagnosed patients infected with CRF01_AE and CRF07_BC virus in Guangdong Province, China. AIDS Res Hum Retroviruses 2012; 28(12):1723-8.

22. Cheng H, Zhang J, Capizzi J, Young NL, Mastro TD. HIV-1 subtype E in Yunnan, China. Lancet 1994; 344(8927):953-4.

23. Han X, Dai D, Zhao B, et al. Genetic and epidemiologic characterization of HIV-1 infection In Liaoning Province, China. J Acquir Immune Defic Syndr 2010; 53 Suppl 1:S27-33.

24. Li L, Lu X, Li H, et al. High genetic diversity of HIV-1 was found in men who have sex with men in Shijiazhuang, China. Infect Genet Evol 2011; 11(6):1487-92.

25. Guo H, Wei JF, Yang H, et al. Rapidly increasing prevalence of HIV and syphilis and HIV-1 subtype characterization among men who have sex with men in Jiangsu, China. Sex Transm Dis 2009; 36(2):120-5.

26. Hemelaar J, Gouws E, Ghys PD, et al. Global trends in molecular epidemiology of HIV-1 during 20002007. AIDS 2011; 25(5):679-89.

27. Xu J, Han X, Reilly KH, Shang H. New features of the HIV epidemic among men who have sex with men in China. Emerg Microbes Infect 2013; 2(7):e45.

28. $\mathrm{Ng} O \mathrm{OT}$, Lin L, Laeyendecker $\mathrm{O}$, et al. Increased rate of $\mathrm{CD} 4+\mathrm{T}$-cell decline and faster time to antiretroviral therapy in HIV-1 subtype CRF01_AE infected seroconverters in Singapore. PLoS One 2011; 6(1):e15738.

29. Chu M, Zhang W, Zhang $X$, et al. HIV-1 CRF01_AE strain is associated with faster HIV/AIDS progression in Jiangsu Province, China. Sci Rep 2017; 7(1):1570.

30. Piyasirisilp S, McCutchan FE, Carr JK, et al. A recent outbreak of human immunodeficiency virus type 1 infection in southern China was initiated by two highly homogeneous, geographically separated strains, circulating recombinant form AE and a novel BC recombinant. $J$ Viro/ 2000; 74(23):11286-95.

31. Yu XF, Chen J, Shao Y, Beyrer C, Lai S. Two subtypes of HIV-1 among injection-drug users in southern China. Lancet 1998; 351(9111):1250. 
32. Su L, Graf M, Zhang Y, et al. Characterization of a virtually full-length human immunodeficiency virus type 1 genome of a prevalent intersubtype (C/B') recombinant strain in China. J Virol 2000; 74(23):11367-76.

33. Jiang YL, Bai WW, Qu FW, et al. Construction and characterization of HIV type 1 CRF07_BC infectious molecular clone from men who have sex with men. J Virol Methods 2016; 229:70-7.

34. Lin PH, Lai CC, Yang JL, et al. Slow immunological progression in HIV-1 CRF07_BC-infected injecting drug users. Emerg Microbes Infect 2013; 2(12):e83.

35. Gui T, Zhao J, Sun C, et al. Genetic Characterization of a Unique Recombinant Originating from CRF55_01B, CRF01_AE, and CRF07_BC in Shenzhen, China. AIDS Res Hum Retroviruses 2015; 31(5):559-63.

36. Wang H, Luo P, Zhu H, et al. Near Full-Length Genomic Characterization of a Novel HIV-1 Unique Recombinant (CRF55_01B/CRF07_BC) from a Malaysian Immigrant Worker in Zhejiang, China. AIDS Res Hum Retroviruses 2017; 33(3):275-8.

37. Cornelissen M, Pasternak AO, Grijsen ML, et al. HIV-1 dual infection is associated with faster CD4+ Tcell decline in a cohort of men with primary HIV infection. Clin Infect Dis 2012; 54(4):539-47.

38. Vasan A, Renjifo B, Hertzmark E, et al. Different rates of disease progression of HIV type 1 infection in Tanzania based on infecting subtype. Clin Infect Dis 2006; 42(6):843-52.

39. Kouyos RD, von Wyl V, Yerly S, et al. Ambiguous nucleotide calls from population-based sequencing of HIV-1 are a marker for viral diversity and the age of infection. Clin Infect Dis 2011; 52(4):532-9.

\section{Tables}

Table 1 Demographic characteristics of the 1792 MSM with recent infection of HIV-1 


\begin{tabular}{|c|c|c|c|c|}
\hline & CRF01_AE & CRF55_01B & CRF07_BC & $P$ \\
\hline & $N=669$ & $N=282$ & $N=841$ & \\
\hline Year & & & & \\
\hline $2005-2008$ & $26(3.9)$ & $9(3.2)$ & $22(2.6)$ & 0.179 \\
\hline 2009 & $47(7.0)$ & $8(2.8)$ & $34(4.0)$ & \\
\hline 2010 & $19(2.8)$ & $8(2.8)$ & $29(3.4)$ & \\
\hline 2011 & $63(9.4)$ & $19(6.7)$ & $68(8.1)$ & \\
\hline 2012 & $124(18.5)$ & $63(22.3)$ & 167(19.9) & \\
\hline 2013 & $163(24.4)$ & $74(26.2)$ & $225(26.8)$ & \\
\hline 2014 & $137(20.5)$ & $60(21.3)$ & $161(19.1)$ & \\
\hline 2015 & $90(13.5)$ & $41(14.5)$ & $135(16.1)$ & \\
\hline Age(year) & & & & \\
\hline$<25$ & $230(34.4)$ & $66(23.4)$ & $288(34.2)$ & $<0.01$ \\
\hline $26-35$ & $292(43.6)$ & $122(43.3)$ & $377(44.8)$ & \\
\hline $36-45$ & $105(15.7)$ & $58(20.6)$ & $136(16.2)$ & \\
\hline$>46$ & $42(6.3)$ & $36(12.8)$ & $40(4.8)$ & \\
\hline Marital status & & & & \\
\hline Unmarried & $542(81.0)$ & 178(63.1) & 654(77.8) & $<0.01$ \\
\hline Married & $88(13.2)$ & $71(25.2)$ & $120(14.3)$ & \\
\hline Divorced or widowed & $29(4.3)$ & $25(8.9)$ & $61(7.3)$ & \\
\hline Unknown & $10(1.5)$ & $8(2.8)$ & $6(0.7)$ & \\
\hline Ethnicity & & & & \\
\hline Han & $31(4.6)$ & $18(6.4)$ & $47(5.6)$ & 0.505 \\
\hline Non-han & 638(95.4) & 264(93.6) & 794(94.4) & \\
\hline Education & & & & \\
\hline Below senior high school & $209(31.2)$ & $114(40.4)$ & $254(30.2)$ & $<0.05$ \\
\hline $\begin{array}{l}\text { Senior high school or technical secondary } \\
\text { school }\end{array}$ & 247(36.9) & $98(34.8)$ & $306(36.4)$ & \\
\hline College or university & $210(31.4)$ & $67(23.8)$ & 275(32.7) & \\
\hline Unknown & $3(0.4)$ & $3(1.1)$ & $6(0.7)$ & \\
\hline
\end{tabular}




\section{Census registration}

Shenzhen resident

$$
\text { 44(9.9) }
$$

19(10.3)

53(9.7)

0.523

Temporary resident

289(64.8)

Floating or unknown

113(25.3)

126(68.1)

381(69.5)

114(20.8)

Note:*Temporary resident indicates the individuals who have household registrations in other regions, and have stayed in Shenzhen city more than six months. Floating population indicates the individuals who have household registrations in other regions, and have stayed in Shenzhen less than six months

Table 2 Estimates of declining rate of CD4 T cell counts from the time of diagnosis to cART initiation

\begin{tabular}{|c|c|c|c|c|c|c|}
\hline \multirow[t]{2}{*}{ HIV-1 Subtype } & \multirow[t]{2}{*}{$\mathrm{N}$} & \multicolumn{3}{|c|}{ Decline rate (cell $/ \mu \mathrm{L})^{1 / 2} /$ year) } & \multicolumn{2}{|c|}{ Decline rate(cell/ $\mu \mathrm{L})^{1 / 2} /$ year $)$} \\
\hline & & Mean $(95 \% \mathrm{Cl})$ & $P$ value & $P$ value & Adjusted mean $(95 \% \mathrm{Cl})$ & $P$ value \\
\hline \multicolumn{7}{|l|}{ CD4 200 350 } \\
\hline CRF55_01B & 62 & 13.6(8.3,18.9) & Ref & & Ref & Ref \\
\hline CRF01_AE & 157 & $23.3(15.9,30.7)$ & $<0.05$ & Ref & $10.3(0.7,19.9)$ & $<0.05$ \\
\hline CRF07_BC & 194 & $11.0(8.4,13.5)$ & 0.391 & $<0.01$ & $-2.7(-12 \cdot 0,6.6)$ & 0.570 \\
\hline \multicolumn{7}{|l|}{ CD4 $\geq 350$} \\
\hline CRF55_01B & 52 & $7.9(3.0,12.8)$ & Ref & & Ref & Ref \\
\hline CRF01_AE & 114 & $6.9(5.0,8.8)$ & 0.702 & Ref & $1.4(-3.5,6.2)$ & 0.587 \\
\hline CRF07_BC & 174 & $9.6(5.6,13.6)$ & 0.597 & 0.226 & $0.7(-4.1,5.5)$ & 0.776 \\
\hline \multicolumn{7}{|l|}{ All $\geq 200$} \\
\hline CRF55_01B & 114 & $11.0(7.3,14.7)$ & Ref & & Ref & Ref \\
\hline CRF01_AE & 271 & $16.4(11.9,20.9)$ & 0.069 & Ref & $6.0(-0.3,12.3)$ & 0.064 \\
\hline CRF07_BC & 368 & $10.3(8.0,12.7)$ & 0.77 & $<0.05$ & $-0.6(-6.7,5.4)$ & 0.837 \\
\hline
\end{tabular}

Note:* The Adjusted mean was estimated with adjustment of age at HIV diagnosis, ethnicity, marital status, and year of HIV diagnosis.

Table 3 Estimates of increasing rate of viral load from the time of diagnosis to cART initiation 


\begin{tabular}{|c|c|c|c|c|c|c|}
\hline & \multirow[t]{2}{*}{$\mathrm{N}$} & \multicolumn{3}{|c|}{$\begin{array}{l}\text { Increase rate of viral load } \\
\left(\log _{10} \text { copies/ml/year) }\right.\end{array}$} & \multicolumn{2}{|l|}{$\begin{array}{l}\text { Increase rate of viral load } \\
\left(\log _{10} \text { copies/ml/year) }\right.\end{array}$} \\
\hline & & Mean $(95 \% \mathrm{Cl})$ & $P$ value & $P$ value & Adjusted mean $(95 \% \mathrm{Cl})$ & $P$ value \\
\hline \multicolumn{7}{|l|}{$\begin{array}{l}\text { CD4 } \\
200 \sim 350\end{array}$} \\
\hline CRF55_01B & 29 & $3.5(1.5,5.5)$ & Ref & & Ref & Ref \\
\hline CRF01_AE & 33 & $1.9(0.9,2.9)$ & 0.150 & Ref & $-1.4(-3.2,0.4)$ & 0.136 \\
\hline CRF07_BC & 27 & $0.9(0.5,1.3)$ & $<0.05$ & $<0.05$ & $-2.6(-4.5,-0.7)$ & $<0.05$ \\
\hline \multicolumn{7}{|l|}{ CD4 $\geq 350$} \\
\hline CRF55_01B & 28 & $0.9(0.6,1.2)$ & Ref & & Ref & Ref \\
\hline CRF01_AE & 21 & $1.4(0.8,2.0)$ & 0.400 & Ref & $0.2(-0.2,0.7)$ & 0.345 \\
\hline CRF07_BC & 28 & $0.7(0.5,0.9)$ & 0.606 & 0.215 & $-0.1(-0.5,0.4)$ & 0.769 \\
\hline \multicolumn{7}{|l|}{ All $\geq 200$} \\
\hline CRF55_01B & 65 & $2.0(1.1,2.9)$ & Ref & & Ref & Ref \\
\hline CRF01_AE & 65 & $1.4(0.8,2.0)$ & 0.324 & Ref & $-0.5(-1.5,0.4)$ & 0.250 \\
\hline CRF07_BC & 69 & $0.7(0.5,0.9)$ & $<0.05$ & $<0.05$ & $-1.2(-2.1,-0.3)$ & $<0.01$ \\
\hline
\end{tabular}

Note:* The Adjusted mean was estimated with adjustment of age, marital status, year of sample and education

\section{Figures}




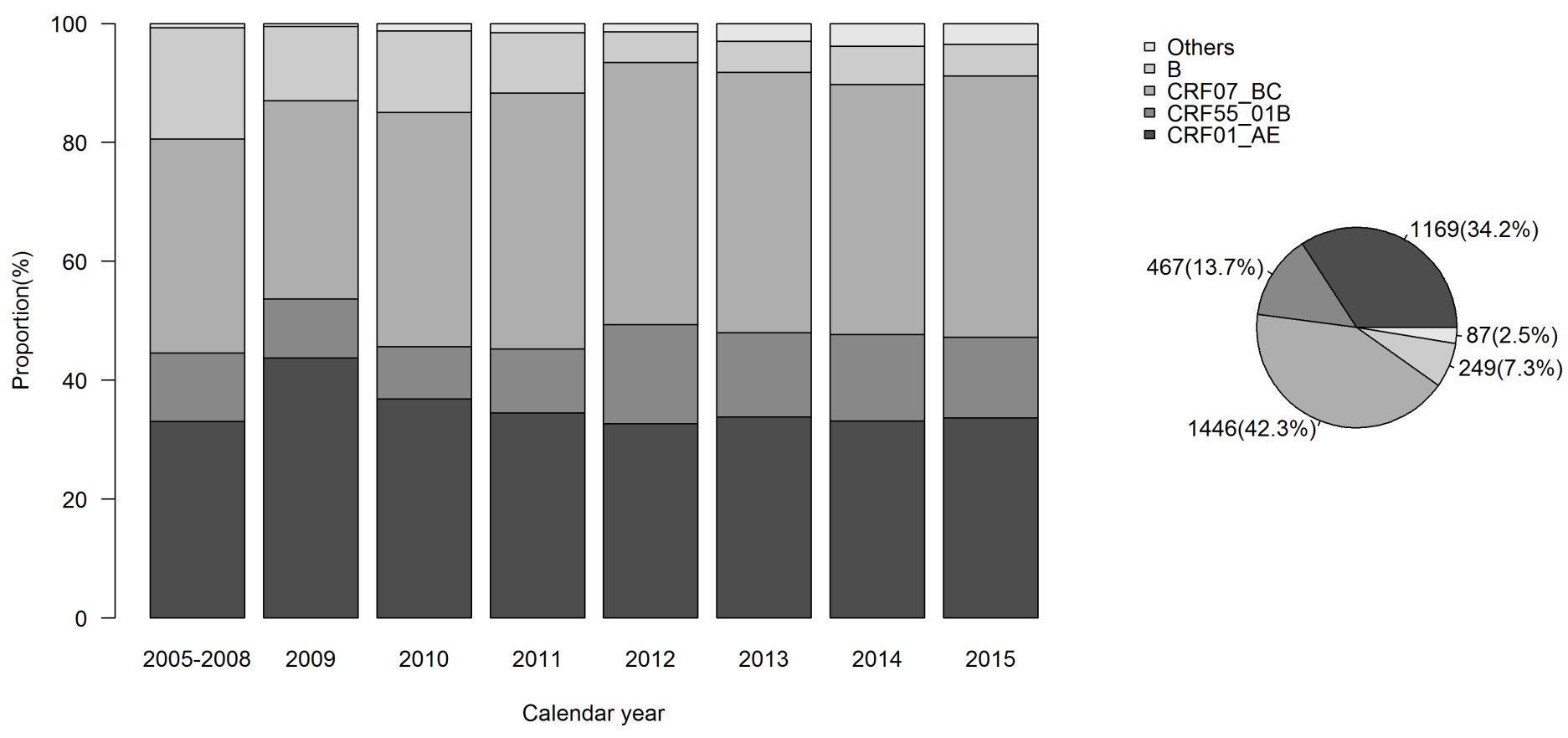

Figure 1

Genotype distribution of HIV-1 among the infected MSM in Shenzhen, China, 2005-2015 

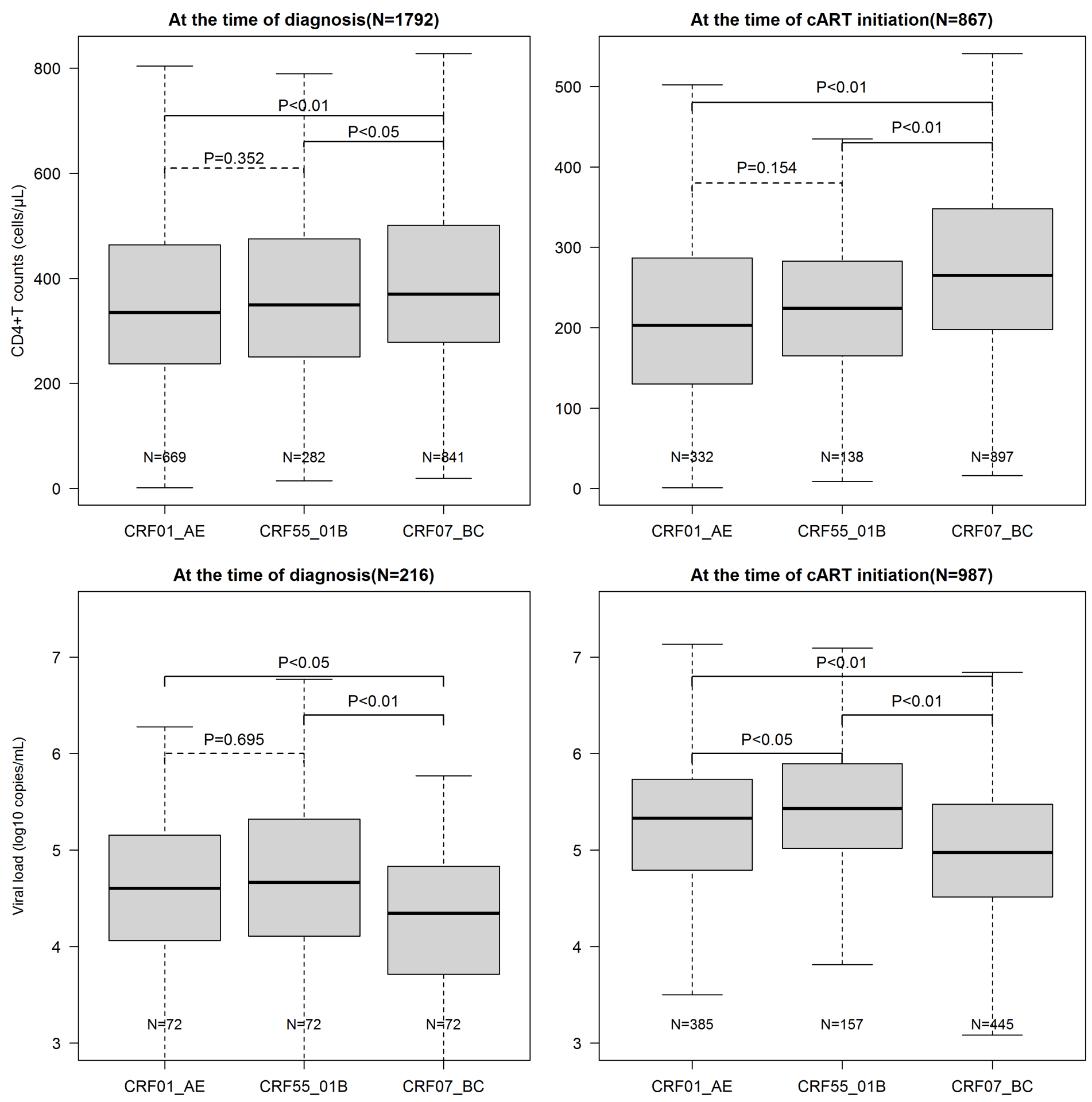

\section{Figure 2}

The CD4 count and plasma HIV RNA load at diagnosis and CART initiation

\section{Supplementary Files}

This is a list of supplementary files associated with this preprint. Click to download. 
- Supplementaltables2021.docx

Page 19/19 